\title{
Secular variation of the Sun's magnetic flux
}

\author{
S. K. Solanki ${ }^{1}$, M. Schüssler ${ }^{1}$, and M. Fligge ${ }^{2}$ \\ 1 Max-Planck-Institut für Aeronomie, Max-Planck-Strasse 2, 37191 Katlenburg-Lindau, Germany \\ e-mail: solanki@linmpi.mpg.de, schuessler@linmpi.mpg.de \\ 2 Institut für Astronomie, ETH-Zentrum, 8092 Zürich, Switzerland
}

Received 18 September 2001 / Accepted 7 December 2001

\begin{abstract}
We present an extension of the model of Solanki et al. (2000) that allows us to reconstruct the time evolution of both the total and the open magnetic flux at the solar surface since 1700. The flux emerging in large active regions is determined using the sunspot number as a proxy, while the flux emergence in small ephemeral regions is described by an extended cycle whose amplitude and length are related to the corresponding sunspot cycle. Both types of regions contribute to the open flux, which is the source of the heliospheric field. The overlap of the activity cycles of ephemeral regions leads to a secular variation of the total cycle-related magnetic flux (active region flux + ephemeral region flux + open flux). The model results indicate that the total surface flux has doubled in the first half of the last century. The evolution of the open flux is in good agreement with the reconstruction by Lockwood et al. (1999).
\end{abstract}

Key words. Sun: magnetic fields - Sun: activity - solar-terrestrial relations

\section{Introduction}

One of the major advances in solar and heliospheric physics of recent years has been the reconstruction of the heliospheric magnetic field (i.e., the Sun's open magnetic flux) from the geomagnetic aa-index by Lockwood et al. (1999). The surprising outcome of this work was the discovery of a secular variation of the heliospheric magnetic field, which is superimposed upon its modulation by the 11-year solar activity cycle: on average, the open flux has doubled since roughly 1900 . The time evolution of the heliospheric field could be reproduced through a simple model by Solanki et al. (2000), whose main assumption is that the open magnetic flux decays rather slowly with an $e$-folding time of about 4 years.

For the understanding of the solar dynamo and of the evolution of solar irradiance it is of far greater importance to know the evolution of the Sun's total magnetic flux (open and closed) than of just the open heliospheric flux. Given the secular variation of the latter, which comprises between roughly $10 \%$ (solar maximum) and up to $30 \%$ (solar minimum) of the unsigned photospheric magnetic flux measured on scales larger than about 10 arcsec (Harvey 1994b; Wang et al. 2000), the question arises whether there is a secular variation of the total flux as well. An indication that this might be the case comes from the observation that the background magnetic flux in the "quiet" network varies much less during the activity cycle than the flux in the active regions (Harvey 1994b) and that there is still considerable magnetic flux present on the solar surface at sunspot minimum. Furthermore, it has been inferred from the observed CaII $\mathrm{H}$ and $\mathrm{K}$ fluxes of Sun-like stars that this background flux may exhibit a significant secular variation (White et al. 1992).

So far there is no physical explanation for such a variation. The concept of a slow decay introduced by Solanki et al. (2000) for the open magnetic flux cannot be extended to the total flux, since there is strong observational evidence that most of the flux appearing on the solar surface disappears within a matter of days to weeks and practically only the open flux in large unipolar regions survives longer than 6 months (Schrijver \& Harvey 1994; Schrijver et al. 1997).

In what follows we present a simple model that leads to a secular variation of the Sun's total cycle-related magnetic flux within the confines of current concepts regarding the solar cycle. It extends the model of Solanki et al. (2000) by including the flux emergence in small ephemeral regions. Their numbers also vary cyclically, with consecutive cycles overlapping each other (Harvey 1992b). The model is based upon the sunspot number record as the most reliable long-term data set directly related to the solar magnetic flux. The model attempts to describe the time evolution of the flux in active regions and 
ephemeral regions as well as of the open heliospheric magnetic flux since 1700 .

\section{The model}

The present model applies exclusively to the cyclically varying magnetic field presumably produced by a largescale dynamo in the deep layers of the convection zone. The magnetic flux emerges at the solar surface in magnetically bipolar regions with a continuous size spectrum (Zwaan \& Harvey 1994). The larger of these are the active regions (AR), which usually contain sunspots and are restricted to roughly $\pm 30^{\circ}$ latitude around the equator. The smaller ephemeral regions (ER) do not form sunspots and have a broader distribution in latitude (Harvey \& Martin 1973; Harvey 1992b, 1993). Any field produced by a potential fast dynamo process on the scale of solar granulation (Petrovay \& Szakaly 1993; Cattaneo 1999) or below is not dealt with here, since there is no reason to assume a secular variation of granulation and, therefore, of the magnetic field it may generate. Consequently, what we call "total flux" in what follows actually comprises only the cycle-related magnetic flux, which may be superimposed upon a time-independent background of small-scale ("internetwork") flux of unknown magnitude.

Dividing the continuous size spectrum of bipolar regions into (large) active regions and (small) ephemeral regions has the advantage of conceptual simplicity: the flux emergence rate in AR can be derived by taking the sunspot number as a "proxy", while the contribution of ER can also be described by a cycle whose amplitude and length we relate for simplicity to the parameters of the corresponding sunspot cycle. For the flux emerging in AR and ER we set up evolution equations. A third equation describes the evolution of the open magnetic flux, which is fed entirely from active and ephemeral regions. Thus the system we need to consider is a set of three ordinary differential equations, which describe the time evolution of each class of magnetic flux as resulting from the corresponding sources and sinks:

$\frac{\mathrm{d} \phi_{\mathrm{act}}}{\mathrm{d} t}=\varepsilon_{\mathrm{act}}(t)-\frac{\phi_{\mathrm{act}}}{\tau_{\mathrm{act}}}-\frac{\phi_{\mathrm{act}}}{\tau_{\mathrm{ta}}}$,

$\frac{\mathrm{d} \phi_{\mathrm{eph}}}{\mathrm{d} t}=\varepsilon_{\mathrm{eph}}(t)-\frac{\phi_{\mathrm{eph}}}{\tau_{\mathrm{eph}}}-\frac{\phi_{\mathrm{eph}}}{\tau_{\mathrm{te}}}$,

$\frac{\mathrm{d} \phi_{\text {open }}}{\mathrm{d} t}=\frac{\phi_{\mathrm{act}}}{\tau_{\mathrm{ta}}}+\frac{\phi_{\mathrm{eph}}}{\tau_{\text {te }}}-\frac{\phi_{\text {open }}}{\tau_{\text {open }}}$.

Here, $\phi_{\text {act }}$ is the (closed) flux that has emerged in active regions, $\phi_{\text {eph }}$ the corresponding flux in ephemeral regions, and $\phi_{\text {open }}$ the open flux. The total flux is given by the sum of these three contributions: $\phi_{\text {tot }}=\phi_{\text {act }}+\phi_{\text {eph }}+$ $\phi_{\text {open }}$. The source terms $\varepsilon_{\text {act }}$ and $\varepsilon_{\text {eph }}$ are the respective flux emergence rates, while $\tau_{\text {act }}, \tau_{\text {eph }}$, and $\tau_{\text {open }}$ are the decay time scales. The decay is due to cancellation with flux of opposite polarity, but otherwise does not need to be specified for the purposes of this model. $\tau_{\text {ta }}$ and $\tau_{\text {te }}$ are the time scales for flux transfer from active and ephemeral regions, respectively, to the open flux.

In order to determine $\varepsilon_{\text {act }}(t)$ we follow Solanki et al. (2000) and write

$\varepsilon_{\mathrm{act}}=c\left(1+\frac{B_{\mathrm{f}} A_{\mathrm{f}}}{B_{\mathrm{s}} A_{\mathrm{s}}}\right) R$,

where $B_{\mathrm{f}}$ is the (spatially averaged) facular magnetic field strength and $A_{\mathrm{f}}$ the facular area, while $B_{\mathrm{s}}$ and $A_{\mathrm{s}}$ are the corresponding quantities for sunspots. $R$ is the sunspot number and $c$ a conversion constant. The term in brackets takes into account that the ratio of magnetic flux in sunspots and in the surrounding facular areas depends on the sunspot number. The factor $B_{\mathrm{f}} / B_{\mathrm{s}} \simeq 0.2$ was not included in the original formulation of Solanki et al. (2000). It reflects the difference in spatially averaged field strength between faculae and sunspots. For the area ratio we have (Chapman et al. 1997; Fligge et al. 1998):

$\frac{A_{\mathrm{f}}}{A_{\mathrm{s}}}=21-0.061 R$.

Note that we have omitted the term proportional to $R^{-1}$ from the original expression as used in Solanki et al. (2000). It is no longer required since here we explicitly account for all cyclically emerging flux in the limit $R \rightarrow 0$ by including the ephemeral regions. Eqs. (4) and (5) show that $\varepsilon_{\text {act }}(t)$ may be written entirely in terms of $R(t)$ and thus can be estimated back to 1700 , or even earlier if the group sunspot number (Hoyt \& Schatten 1998) is employed. Using the value $\varepsilon_{\text {act, } \max }=2.3 \times 10^{24} \mathrm{Mx} \mathrm{yr}^{-1}$ given by Schrijver \& Harvey (1994) for the emergence rate during the maximum of sunspot cycle 21 together with the corresponding sunspot number of $R=150$, we obtain from Eqs. (4) and (5) that $c=4.5 \times 10^{21} \mathrm{Mx} \mathrm{yr}^{-1}$.

We estimate the decay time of the AR flux, $\tau_{\text {act }}$, by assuming an instantaneous balance of flux emergence and decay during the maximum of cycle $21: \phi_{\text {act, } \max }=$ $\tau_{\text {act }} \cdot \varepsilon_{\text {act,max }}$. In doing so, we neglect the rate of transfer of closed active region flux to open flux described by the transfer time $\tau_{\text {ta }}$. This is justified a posteriori by the resulting relation $\tau_{\text {act }} \ll \tau_{\text {ta }} \simeq 10$ yr (see Sect. 3.3). Using the values given by Schrijver \& Harvey (1994), $\phi_{\text {act, } \max }=5 \times 10^{23} \mathrm{Mx}$ and $\varepsilon_{\text {act, } \max }=2.3 \times 10^{24} \mathrm{Mx} \mathrm{yr}^{-1}$, we obtain $\tau_{\text {act }}=0.22 \mathrm{yr}$. For the corresponding decay time of the ER flux we take the value $\tau_{\text {eph }}=14 \mathrm{~h}$ given by Hagenaar (2001). Since both decay times, $\tau_{\text {act }}$ and $\tau_{\text {eph }}$, are small compared to the cycle period of about 11 years, the fluxes $\phi_{\text {act }}$ and $\phi_{\text {eph }}$ are well approximated by assuming an instantaneous balance between the source and sink terms on the right-hand sides of Eqs. (1) and (2):

$$
\begin{aligned}
& \phi_{\mathrm{act}}=\varepsilon_{\mathrm{act}}\left(\frac{\tau_{\mathrm{act}} \tau_{\mathrm{ta}}}{\tau_{\mathrm{act}}+\tau_{\mathrm{ta}}}\right), \\
& \phi_{\mathrm{eph}}=\varepsilon_{\mathrm{eph}}\left(\frac{\tau_{\mathrm{eph}} \tau_{\mathrm{te}}}{\tau_{\mathrm{eph}}+\tau_{\mathrm{te}}}\right) .
\end{aligned}
$$




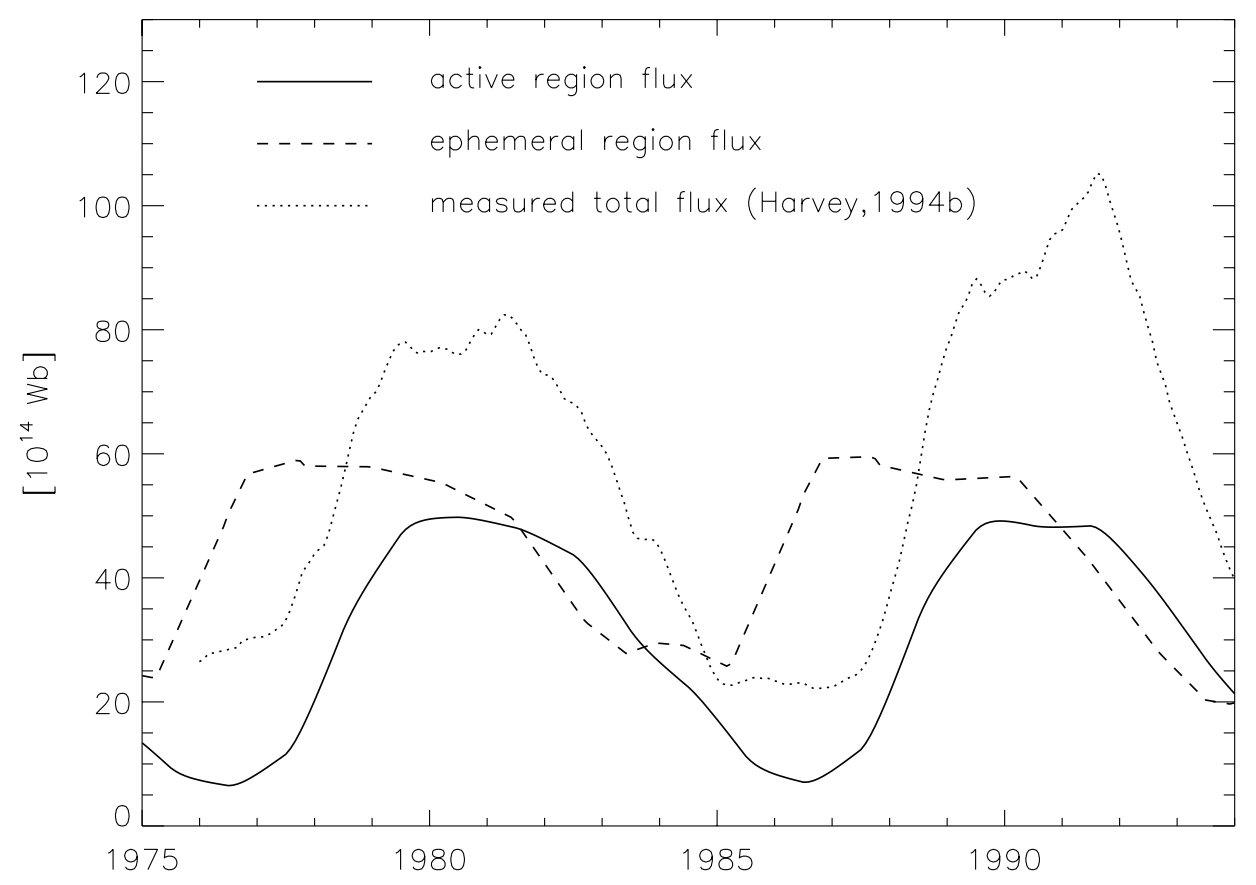

Fig. 1. Time evolution of measured total flux (Harvey 1994b, dotted line) together with the modeled active region (full line) and ephemeral region fluxes (dashed line). The assumed lead time for the ephemeral region cycle is $\tau_{x}=2.5 \mathrm{yr}$.

Equation (3) for the open flux then becomes

$\frac{\mathrm{d} \phi_{\text {open }}}{\mathrm{d} t}=\frac{\varepsilon_{\mathrm{act}} \tau_{\mathrm{act}}}{\tau_{\mathrm{act}}+\tau_{\mathrm{ta}}}+\frac{\varepsilon_{\mathrm{eph}} \tau_{\mathrm{eph}}}{\tau_{\mathrm{eph}}+\tau_{\mathrm{te}}}-\frac{\phi_{\text {open }}}{\tau_{\text {open }}}$.

In order to specify $\varepsilon_{\mathrm{eph}}(t)$, we make the basic assumption that ER are products of the same dynamo mechanism as the larger AR, representing their continuation to smaller scales. This implies that $\varepsilon_{\text {eph }}(t)$ should vary cyclically and in a fixed phase relation with $\varepsilon_{\text {act }}(t)$, although not necessarily with the same cycle length. The functional form of $\varepsilon_{\text {eph }}(t)$ can be constrained with the help of the data presented by Harvey $(1993,1994 b)$, which indicate that $\varepsilon_{\text {eph }}$ runs somewhat ahead of $\varepsilon_{\text {act }}$ in phase: ER belonging to a given cycle (as distinguished, e.g., according to latitude) start emerging 2-3 years before the first sunspots appear. This leads to the situation that, for some time near the end of a sunspot cycle, we have an overlap of magnetic flux emergence in ER from the old and from the new cycle. Since such an overlap is almost non-existent for the AR, the cycle variation of ER flux ("quiet network flux") becomes much smaller than the variation of AR flux (Harvey 1994b). The simplest prescription for $\varepsilon_{\mathrm{eph}, i}(t)$ (emergence rate for the $i$ th cycle) is to stretch the corresponding sunspot cycle of length $\tau_{i}=t_{i+1}-t_{i}$ (where $t_{i}$ and $t_{i+1}$ are the epochs of the adjacent sunspot minima) to a length $\tau_{i}+\tau_{x}$, so that the cycle of ER starts a time interval $\tau_{x}$ earlier than the corresponding sunspot cycle:

$\varepsilon_{\mathrm{eph}, i}(t)=X \cdot \varepsilon_{\mathrm{act}, i}\left(t^{\prime}\right)$,

with

$t^{\prime}=t_{i}+\left(t-t_{i}+\tau_{x}\right) \cdot \frac{\tau_{i}}{\tau_{x}+\tau_{i}}$, where $t_{i}-\tau_{x} \leq t \leq t_{i+1}$. Since the cycles overlap, we have to sum over multiple cycles to obtain the total flux emergence rate in ER: $\varepsilon_{\text {eph }}(t)=\sum_{i} \varepsilon_{\text {eph }, i}(t)$. In order to avoid jumps, a smooth transition to zero (over one year) is added to $\varepsilon_{\mathrm{eph}, i}(t)$ at the edges of the corresponding time interval. The (time-independent) amplitude factor $X$ can be estimated by using the emergence rate $\varepsilon_{\text {eph,min }}=1.8 \times 10^{26} \mathrm{Mx} \mathrm{yr}^{-1}$ of ER during the last solar minimum (Hagenaar 2001) and assuming that the emergence rate at activity maximum is larger by a factor 2 (Harvey 1993): $X=2 \cdot \varepsilon_{\text {eph,min }} / \varepsilon_{\text {act,max }} \simeq 160$. We may either take the lead time $\tau_{x} \simeq 2 \ldots 3$ yr to be the same for all cycles or assume that the (forward) extension of the ephemeral region cycle is a fixed fraction of the current sunspot cycle length, so that $\tau_{x}=y \tau_{i}$ with $y \simeq 0.2 \ldots 0.3$. It turns out that the results are almost independent of this choice as long as values are used that are compatible with the observations of Harvey (1993).

The transfer times from AR/ER flux to open flux, $\tau_{\text {ta }}$ and $\tau_{\text {te }}$, are of the order of $10 \mathrm{yr}$ and more, so that they do not significantly influence the evolution of $\phi_{\text {act }}$ and $\phi_{\text {eph }}$. The choice of these parameters and the decay time $\tau_{\text {open }}$ affects only the open flux (see Sect. 3.3).

\section{Results}

\subsection{Comparison with measured fluxes}

The validity of our model is tested by comparing with the time evolution of the total magnetic flux in cycles 21 and 22 (Harvey 1994b) derived from synoptic charts constructed from Kitt Peak magnetograms (Harvey 1992a). Figure 1 shows these data (dotted line) together with the fluxes in AR (full line) and ER (dashed line) as resulting 


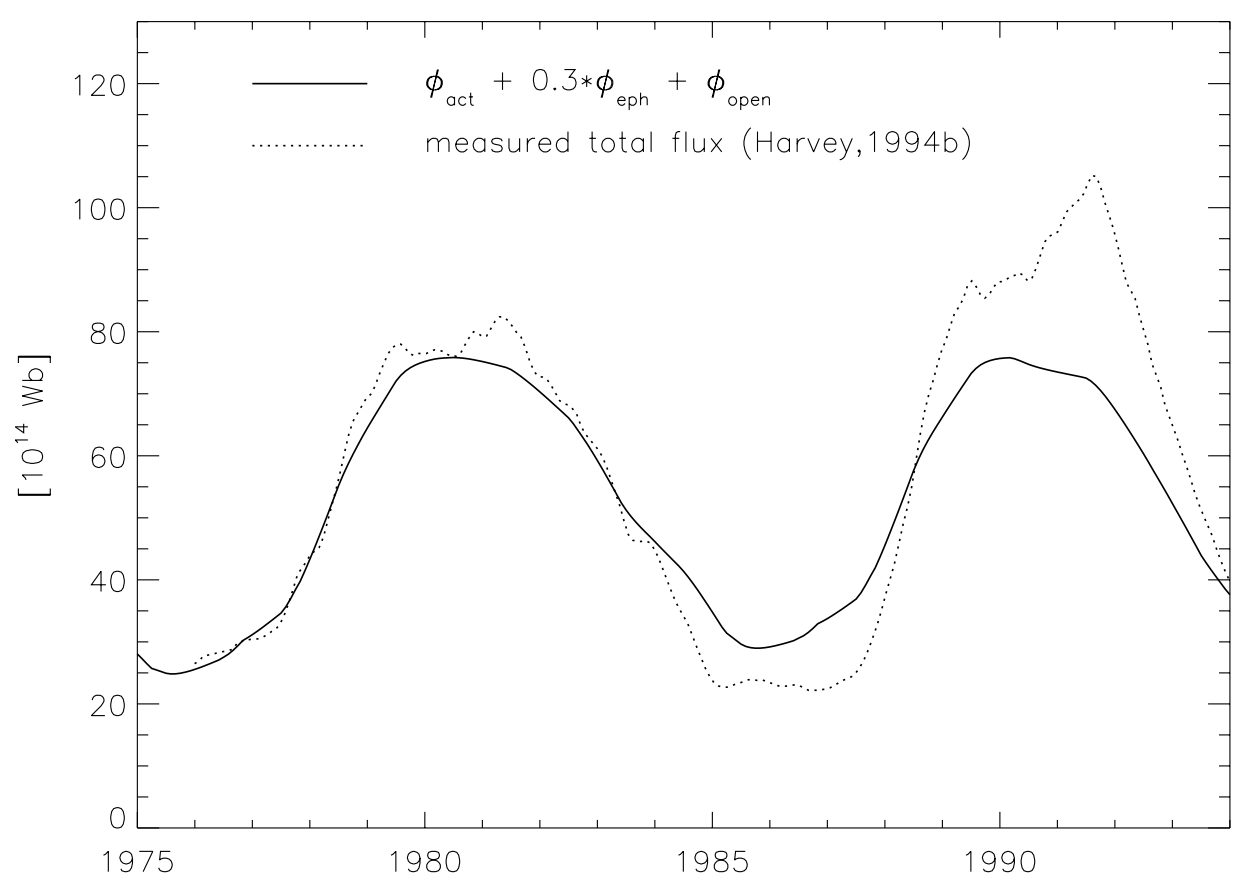

Fig. 2. Time evolution of measured total flux (Harvey 1994b, dotted line) in comparison with the quantity $\phi_{\text {act }}+0.3 \cdot \phi_{\mathrm{eph}}+\phi_{\mathrm{open}}$ (full line) as determined from the model for the same parameters as in Fig. 1.

from our model with $\tau_{x}=2.5 \mathrm{yr}$. All other parameters are chosen as described in the previous section. The ER cycle is longer than the AR (sunspot) cycle and shifted forward in time. Since the ER cycles overlap, the ER flux varies by only a factor 2 from from minimum to maximum, while the minimum flux in ER is reached about $1.5 \mathrm{yr}$ before sunspot minimum. Both of these properties are in accordance with the observations of Harvey (1994b).

Comparison with the corresponding separated curves for the flux in AR and in "quiet" regions (Harvey 1994a,b) indicates that the synoptic charts, which have a relatively low spatial resolution, capture only about $30 \%$ of the ER flux. Possible reasons for this uncomplete detection are the spatial averaging over regions of mixed polarity and the weakening of spectral lines in the hot magnetic elements forming the network. Independent evidence that only a fraction of the magnetic flux is detected comes from the fact that chromospheric and coronal proxies of magnetic flux (such as He I 10830) exhibit a much larger quiet sun contribution than suggested by the associated magnetic flux measurements (e.g., Harvey 1994a). Furthermore, the hysteresis between the Kitt Peak magnetic index and the frequency shifts of intermediate degree $p$-modes reveals that the $p$-modes react to changes associated with the magnetic field earlier than the Kitt Peak magnetograms (Tripathy et al. 2001). Since the $p$ mode shifts follow the amount of magnetic flux (Goldreich et al. 1991), this suggests that a part of the early rise in magnetic flux, due to ephemeral regions, is missed by the magnetograms.

We take into account the incomplete detection of ER flux and compare in Fig. 2 the quantity $\phi_{\text {act }}+0.3 \cdot \phi_{\text {eph }}+$ $\phi_{\text {open }}$ (full line) with the measured total flux. The model matches the order of magnitude of the flux variations and the cycle length rather well but, being based only on sunspot number data, the model must fail to reproduce the difference in the maximum fluxes of cycles 21 and 22 and shows a somewhat less flat minimum between ${ }^{1}$. This could be due to a different ratio of facular to sunspot flux for the two cycles, which may exhibit more subtle variations than those described by Eqs. (4) and (5). The difference could also arise if the factor 0.3 for the detection efficiency of ER does not apply to both cycles because of a change in the distribution of ER sizes; in this case it would have implications for the comparison but not for the model itself.

\subsection{Reconstruction of the magnetic fluxes}

Having confirmed the validity of our model, we may proceed to reconstructing the AR, ER, and total fluxes since 1700. We integrate the evolution equations for the fluxes, Eqs. (1)-(3), forward in time with a step size of one month. Owing to the small decay time of only $14 \mathrm{~h}$ for $\phi_{\mathrm{eph}}$, we can use Eq. (7) to determine the ER flux. The resulting time evolution of the various fluxes is shown in Fig. 3. The total flux contains also the open flux, for which we took a reconstruction providing a good fit to the data of Lockwood et al. (1999) and comprising between about 10\% (solar maximum) and $30 \%$ (solar minimum) of the measured

${ }^{1}$ A recent re-calibration of the NSO Kitt Peak magnetograph data indicates that the maxima of cycles 21 and 22 were more similar to each other than shown in Fig. 2, so that the reconstruction may in fact be in better agreement with the data (J.W. Harvey, private communication). 

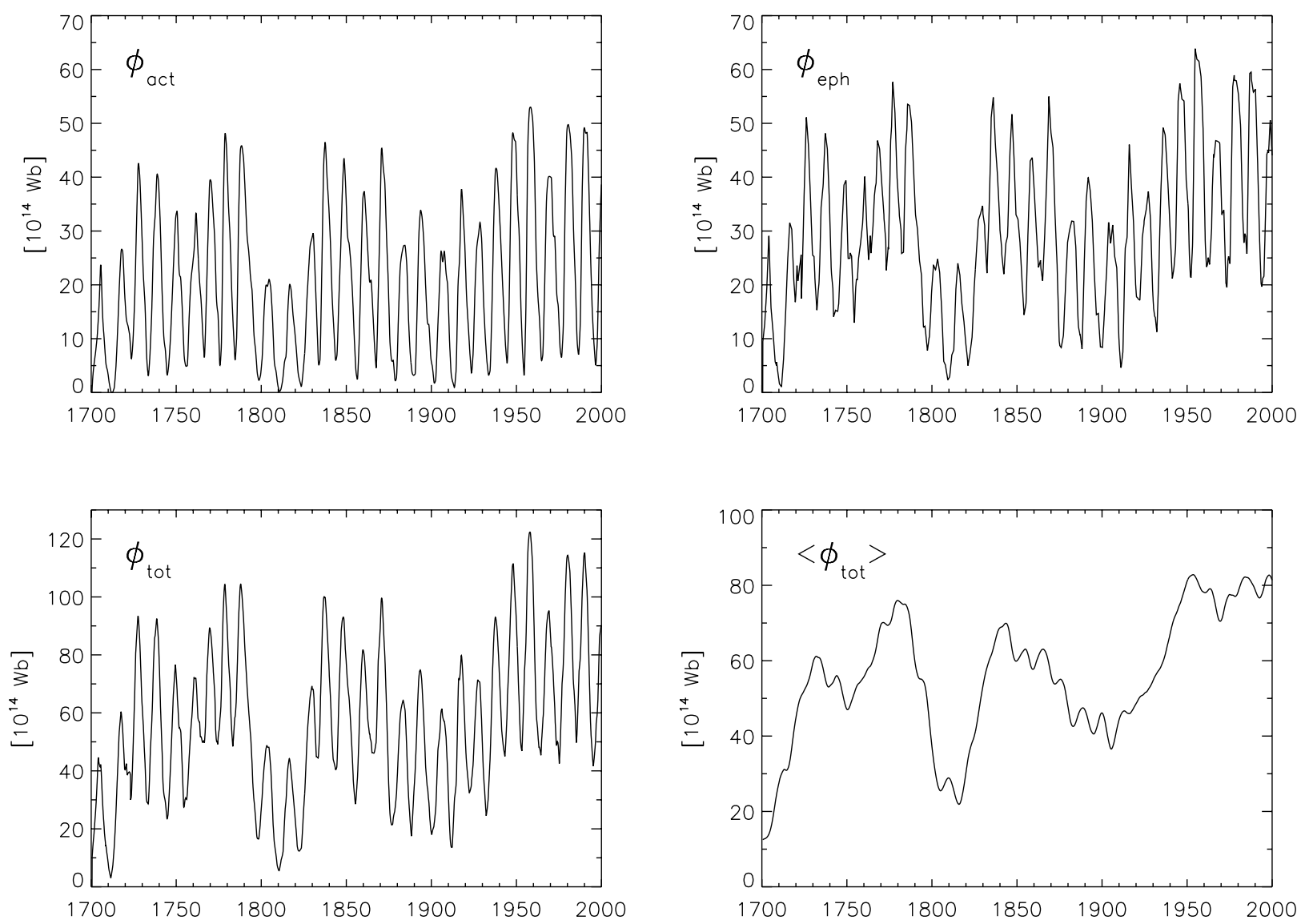

Fig. 3. Reconstructed magnetic fluxes since 1700: active regions (upper left), ephemeral regions (upper right), total flux (lower left), and 20-year running mean of total flux (lower right). On average, the total flux has about doubled in the first half of the last century.

total flux. More details about the open flux are given in the following subsection.

Due to the overlapping cycles of the ER, Fig. 3 shows a clear secular variation of both the ER flux and the total flux, best visible in the lower right panel showing a 20-year running mean of the total flux. The Dalton minimum around 1800 leads to a drop in total flux by more than a factor 3 while the flux, on average, has doubled between roughly 1900 and 1950 .

\subsection{Open flux}

The time evolution of the open flux has successfully been reconstructed by Solanki et al. (2000) purely on the basis of the AR flux. We can reproduce these results by using Eq. (8) with the following parameters: $\tau_{\text {eph }}=0$, $\tau_{\text {act }}=0.22 \mathrm{yr}, \tau_{\mathrm{ta}}=12 \mathrm{yr}$, and $\tau_{\text {open }}=4 \mathrm{yr}$. The new feature of the extended model presented here is the inclusion of the ephemeral regions. In principle, the overlap of ER cycles could contribute to the secular trend of the open flux and possibly permit a smaller value of the decay time, $\tau_{\text {open }}$. However, the contribution of ER to the open flux is rather uncertain. Although ER comprise a significant fraction of the total flux on the solar surface and dominate around sunspot minimum, their individual dipole moments are smaller and their orientations are more randomly distributed than those of AR. Harvey (1994b) found that in cycle 21 the contribution of the ER to the axial dipole moment of the Sun was about a factor of 6 smaller than that of the AR and, surprisingly, of opposite sign.

In order to estimate the potential effect of ER on the open flux, we use the factor 6 indicated by the analysis of Harvey (1994a) and write $\tau_{\text {te }}=6 \cdot \tau_{\text {ta }}=72 \mathrm{yr}$. If we assume that the effects of ER and AR on the open flux have the same sign, we obtain a good agreement with the reconstruction of Lockwood et al. (1999) with a smaller decay time of the open flux than used by Solanki et al. (2000): $\tau_{\text {open }}=3 \mathrm{yr}$ instead of $4 \mathrm{yr}$ without ER (see Fig. 4 ). However, if the effects of ER and AR are always of opposite sign as found by Harvey (1994b) for cycle 21, we need $\tau_{\text {open }} \simeq 5$ yr and obtain a noticeable phase shift between the modeled open flux and the reconstruction of Lockwood et al. (1999). Consequently, in the framework of our model it seems unlikely that the effects of ER and $\mathrm{AR}$ on the open flux are of opposite sign for more than a minority of solar cycles.

The potential relevance of ER for the open flux is emphasized by the indications from the ${ }^{10} \mathrm{Be}$ record that the cyclic solar activity continued throughout the Maunder minimum (Beer et al. 1998). The "cosmogenic" 


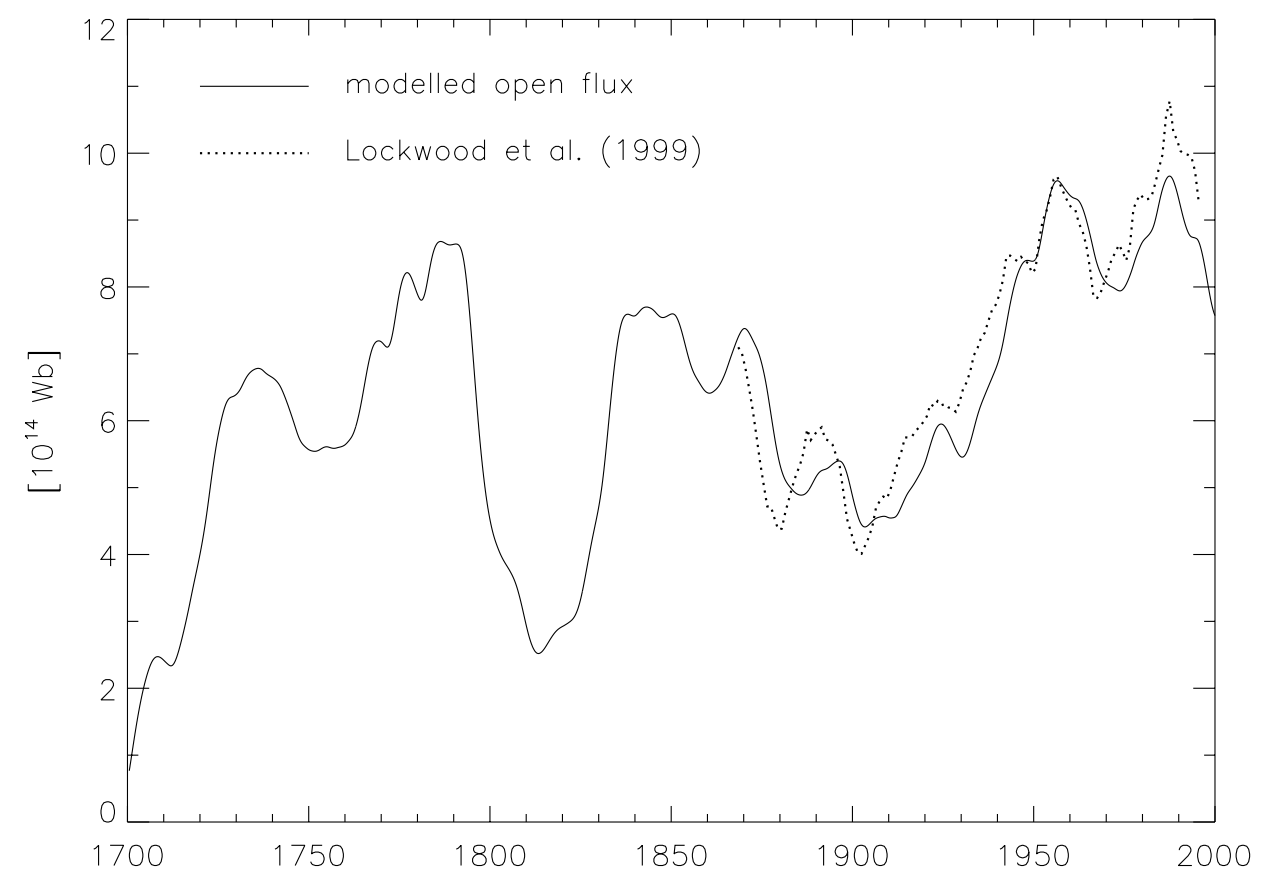

Fig. 4. Full line: 11-yr running mean of the open solar flux obtained from our model including ephemeral regions. A decay time of $\tau_{\text {open }}=3$ yr has been employed. Dotted line: 11-yr running mean of the reconstructed heliospheric flux from Lockwood et al. (1999).

isotope ${ }^{10} \mathrm{Be}$ is produced in the terrestrial atmosphere by cosmic rays (e.g., see Beer 2000), which are modulated by the variation of the heliospheric magnetic field (the open solar flux). Since sunspots were almost absent during the Maunder minimum, the variability of the open flux during that time, if real, was probably caused by ER. A strong increase of the ${ }^{10} \mathrm{Be}$ production rate, i.e. a sharp drop of the amount of open flux, is only seen near the end of the Maunder minimum. Interestingly, a very good fit to the data of Lockwood et al. (1999) showing no relative phase shift is achieved by taking exclusively the ER as sources of the open flux and setting $\tau_{\text {te }}=12 \mathrm{yr}$.

\section{Concluding remarks}

The extension of the model of Solanki et al. (2000) to include the magnetic flux emerging in ephemeral regions permits the reconstruction of the time evolution of both the open source flux and the total (cycle-related) magnetic flux at the solar surface back to the beginning of the systematic sunspot record at the end of the Maunder minimum. Clearly our model represents a drastic simplification of the processes that determine the evolution of magnetic flux on the solar surface, so that we cannot expect more than a rough agreement between the reconstruction from the model and the actual flux values (see Figs. 1 and 2). Nevertheless, the model results indicate that the overlap of the activity cycles of ephemeral regions causes a secular modulation of the total flux that reflects the long-term variation of solar activity. In particular, the model suggests that the Dalton minimum around 1800 led to a drop by about a factor 3 , while the total flux doubled in the first half of the 20th century. Such modulation could affect the variability of solar irradiance and thus have an impact on the terrestrial climate (e.g., Solanki \& Fligge 2000). Our work may therefore provide a theoretical basis for secular irradiance changes, whose existence has previously been inferred only indirectly based on the comparison with other stars (e.g., White et al. 1992). A similar secular variation of the open flux and heliospheric magnetic field affects the flux of cosmic rays hitting the terrestrial atmosphere. Climatic effects of such a variation have also been suggested (Marsh \& Svensmark 2000).

Acknowledgements. The fundamental and thorough work of Karen Harvey has provided the inspiration for the present investigation. We are grateful to Karel Schrijver for drawing our attention to the possibility of a secular variation caused by the overlapping cycles of ephemeral regions.

\section{References}

Beer, J. 2000, in Solar Variability and Climate, ed. E. Friis-Christensen, C. Fröhlich, J. Haigh, M. Schüssler, \& R. von Steiger (Dordrecht: Kluwer Academic Publishers), 53

Beer, J., Tobias, S., \& Weiss, N. 1998, Sol. Phys., 181, 237

Cattaneo, F. 1999, ApJ, 515, L39

Chapman, G. A., Cookson, A. M., \& Dobias, J. J. 1997, ApJ, 482, 541

Fligge, M., Solanki, S. K., Unruh, Y. C., Fröhlich, C., \& Wehrli, C. 1998, A\&A, 335, 709

Goldreich, P., Murray, N., Willette, G., \& Kumar, P. 1991, ApJ, 370, 752

Hagenaar, H. J. 2001, ApJ, 555, 448

Harvey, K. L. 1992a, in Proceedings of Solar Electromagnetic Radiation Study for Cycle 22, ed. R. Donnelly, NOAA/ERL/SEL, Boulder, CO., 113 
Harvey, K. L. 1992b, in The Solar Cycle, ed. K. L. Harvey (San Francisco: Astronomical Society of the Pacific), ASP Conf. Ser., 27, 335

Harvey, K. L. 1993, Ph.D. Thesis, University of Utrecht, The Netherlands

Harvey, K. L. 1994a, in The Sun as a Variable Star: Solar and Stellar Irradiance Variations, ed. J. M. Pap, C. Fröhlich, H. S. Hudson, \& S. K. Solanki (Cambridge: Cambridge Univ. Press), 217

Harvey, K. L. 1994b, in Solar Surface Magnetism, ed. R. J. Rutten, \& C. J. Schrijver (Dordrecht: Kluwer), 347

Harvey, K. L., \& Martin, S. F. 1973, Sol. Phys., 32, 389

Hoyt, D. V., \& Schatten, K. H. 1998, Sol. Phys., 179, 189

Lockwood, M., Stamper, R., \& Wild, M. N. 1999, Nature, 399, 437

Marsh, N., \& Svensmark, H. 2000, in Solar Variability and Climate, ed. E. Friis-Christensen, C. Fröhlich, J. Haigh, M. Schüssler, \& R. von Steiger (Dordrecht: Kluwer Academic Publishers), 215
Petrovay, K., \& Szakaly, G. 1993, A\&A, 274, 543

Schrijver, C. J., \& Harvey, K. L. 1994, Sol. Phys., 150, 1

Schrijver, C. J., Title, A. M., van Ballegooijen, A. A., Hagenaar, H. J., \& Shine, R. A. 1997, ApJ, 487, 424

Solanki, S. K., \& Fligge, M. 2000, in Solar Cycle and Terrestrial Climate, ed. A. Wilson, ESA SP-463, 51

Solanki, S. K., Schüssler, M., \& Fligge, M. 2000, Nature, 408, 445

Tripathy, S. C., Kumar, B., Jain, K., \& Bhatnagar, A. 2001, Sol. Phys., 200, 3

Wang, Y.-M., Lean, J., \& Sheeley, N. R. 2000, Geophys. Res. Lett., 27, 505

White, O. R., Skumanich, A., Lean, J., Livingston, W. C., \& Keil, S. L. 1992, Publ. Astron. Soc. Pac., 104, 1139

Zwaan, C., \& Harvey, K. L. 1994, in Solar Magnetic Fields, ed. M. Schüssler, \& W. Schmidt (Cambridge: Cambridge University Press), 27 